\title{
Environmental sample characteristics and herd size associated with decreased herd-level prevalence of Mycobacterium avium ssp. paratuberculosis
}

\author{
Caroline S. Corbett, S. Ali Naqvi, Jeroen De Buck, Uliana Kanevets, John P. Kastelic, \\ and Herman W. Barkema ${ }^{1}$ \\ Department of Production Animal Health, Faculty of Veterinary Medicine, University of Calgary, Calgary, AB, Canada, T2N 4N1
}

\begin{abstract}
Environmental sampling is an effective method for estimating regional dairy herd-level prevalence of infection with Mycobacterium avium ssp. paratuberculosis (MAP). However, factors affecting prevalence estimates based on environmental samples are not known. The objective was to determine whether odds of environmental samples collected on farm changed culture status over 2 sampling times and if changes were specific for location and type of housing (freestall, tiestall, or loose housing), the sample collected (i.e., manure of lactating, dry, or sick cows; namely, cow group), and effects of herd size. In 2012-2013 [sampling 1 (S1)] and 2015-2017 [sampling 2 (S2)], 6 environmental samples were collected and cultured for MAP from all $167(99 \%)$ and 160 (95\%) farms, respectively, in the province of Saskatchewan, Canada. Only the 148 dairy farms sampled at both sampling periods were included in the analysis. A mixed effects logistic regression was used to determine whether differences between sampling periods were associated with herd size and sample characteristics (cow group contributing to environmental sample, type of housing, and location). In S1 and $\mathrm{S} 2,55$ and $34 \%$, respectively, of farms had at least 1 MAP-positive environmental sample. Correcting for sensitivity of environmental sampling, estimated true prevalence in S1 and S2 was 79 and $48 \%$, respectively. Herds with $>200$ cows were more often MAP-positive than herds with $<51$ cows in both S1 and S2. The percentage of positive samples was lower in S2 compared with S1 for all sampled areas, cow groups contributing to samples, types of housing where samples were collected, and herd size categories. However, samples collected from dry cow areas had the largest decrease in MAP-positive samples in S2 compared with all other
\end{abstract}

Received February 27, 2018.

Accepted May 17, 2018.

${ }^{1}$ Corresponding author: barkema@ucalgary.ca cow group samples. Herds that were MAP-negative in S1 with a herd size 51 to 100 or 101 to 150 were more likely to stay MAP-negative, whereas MAP-positive herds with $>200$ cows more frequently stayed MAPpositive. No difference was observed in the odds of a sample being MAP-positive among housing types or location of sample collection in both sample periods. Of all farms sampled, 104 (70\%) did not change status from S1 to S2. In conclusion, when herd-level MAP prevalence decreased over the 3-yr interval, the change in prevalence differed among herd size categories and was larger in samples from dry cow areas. It was, however, not specific to other characteristics of environmental samples collected.

Key words: paratuberculosis, environmental sample, Johne's disease, herd size, Saskatchewan

\section{INTRODUCTION}

Johne's disease (JD), a chronic enteritis caused by the bacterium Mycobacterium avium ssp. paratuberculosis (MAP), has an adverse economic impact on the dairy industry worldwide due to decreased milk production, increased risk of culling, and decreased slaughter value (Tiwari et al., 2006; Lombard, 2011; Smith et al., 2017). No cure or effective vaccine for prevention of MAP infection is available; therefore, control programs are primarily based on decreasing risk of new infections within a dairy herd (Kalis et al., 2001; McKenna et al., 2006). Canadian JD control initiatives rely on detection of MAP-positive herds and subsequent risk assessments, resulting in changes to management practices to decrease new infections within a herd (Wolf et al., 2014b). Following detection of a positive herd, producers can opt for individual cow testing to remove infectious cattle; however, detection of these cattle is difficult due to the prolonged incubation period, unreliable diagnostics, and variability of immune and symptomatic responses (Marcé et al., 2010; Barkema et al., 2018). Due to high variability among diagnostic tests in test characteristics, prevalence estimates can vary sub- 
stantially; therefore, test sensitivities and specificities must be considered when estimating true prevalence (Barkema et al., 2018).

True cow-level prevalence estimates are difficult to estimate, as diagnostic tests rely on detecting latent and varying immune responses of cattle, or detection of the pathogen, which is intermittently shed in milk and feces. Infected cattle can be identified as negative if sampled at a time of no shedding or before immune responses develop, and this may result in low-prevalence herds being categorized as negative, despite MAP-infected cattle being present (Raizman et al., 2007; Donat et al., 2015). Fecal shedding can be intermittent and extent of shedding is highly variable (Mitchell et al., 2015), which has large consequences for transmission of MAP, as the primary route of infection is fecal oral. However, due to survivability of MAP in the environment for prolonged intervals (Whittington et al., 2004), environmental samples are a cost-effective and reliable method for detection of MAP-positive herds (Berghaus et al., 2006), and are currently used in control programs in the United States and Canada (Whitlock, 2010; Wolf et al., 2014b).

The most common sampling method protocol for environmental samples requires 6 samples to be collected from various locations on a dairy farm (Berghaus et al., 2006). The type of sample collected is important, as sample characteristics affect the likelihood of a MAP-positive result (Wolf et al., 2015). For example, environmental samples from the lactating cow area are more likely to be positive than those from sick/calving pens or dry cow pens; furthermore, samples collected from locations where manure from several cows accumulate (e.g., alley ways or lagoons) are more likely to be positive than bedded packs or manure piles (Wolf et al., 2015). These sample-type specific characteristics of environmental samples can be grouped based on cows contributing to the sample (cow group; i.e., lactating, dry, sick, and so on), type of pen that cows are housed in (housing type; i.e., freestall, tiestall, loose housing, and so on) and location collected (location type; i.e., alley, gutter, bedding pack, and so on). Additionally, larger herds are more likely to have MAP-positive samples and have higher within-herd prevalence than smaller herds (Wells and Wagner, 2000); however, there is no evidence that herd size affects sample-type-specific results (Wolf et al., 2015).

Accurate prevalence estimates are essential for control, surveillance, and monitoring effectiveness of a control program over time (Barkema et al., 2018). In long-term studies, herd MAP prevalence estimates decrease over time when control programs are in place (Collins et al., 2010; Sorge et al., 2011). Most programs used milk or serum antibody ELISA to estimate herd prevalence, although it is unknown how or if herd MAP prevalence estimates based on environmental samples are associated with characteristics of environmental samples (cow group, housing, and location) and interact to skew apparent changes in prevalence at various time points, or if they change in association with prevalence estimates. The prevalence of MAP-infected herds based on environmental samples in Saskatchewan, Canada, has been reported (Wolf et al., 2014a). However, stability of herd infection status and associations with herd size following implementation of a control program has not been documented. The objective was to determine if odds of environmental samples collected on-farm changing MAP culture status over the 2 sampling times were specific for location and type of housing (freestall, tiestall, or loose housing) the sample has been collected, whether it included manure of lactating, dry, or sick cows (cow group), and whether it was associated with herd size.

\section{MATERIALS AND METHODS}

\section{Herds}

Environmental samples were collected from all 167 and 160 dairy farms in the province of Saskatchewan in the first and second samplings, respectively, as part of the Saskatchewan JD surveillance program of the Saskatchewan Ministry of Agriculture (Regina, SK, Canada) and SaskMilk (Regina, SK, Canada), the Saskatchewan dairy producer marketing board. Only farms sampled at both sampling periods $(\mathrm{n}=148)$ were included in the analysis. Farms were visited once by a SaskMilk field technician (Regina, SK, Canada) between August 2012 and October 2013 (sampling period 1; S1), and a second time between September 2015 and February 2017 (sampling period 2; S2) by the same technician. Mean interval between sampling periods was 3.5 yr (17 farms sampled after 2 yr, 59 farms after 3 yr, 51 farms after 4 yr, and 21 farms after $5 \mathrm{yr}$ ). Herd size was categorized into 5 categories: $<51$, $51-100,101-150,151-200$, and $>200$ cows. Herd size information was collected at S1 and applied to farms at S2. Following the first environmental sample collection in S1, MAP-positive farms were offered an option to enroll in whole-herd testing (individual serum ELISA, individual fecal testing, or both), along with completion of a risk assessment by the herd veterinarian for improvement of management practices. Veterinarians were trained to identify MAP transmission-specific risk factors and make suggestions within dairy producers capabilities for improvement. Any suggested changes to improve JD control were individualized per farm, specific to the risk assessment performed by the veteri- 
narian, and implemented based on the discretion of the dairy producer. Unfortunately, no information on these actions or results of individual whole-herds testing were available for analysis, due to strict privacy rules associated with the JD control program.

\section{Sample Collection}

Sample collection was done as described (Wolf et al., 2014a). Briefly, the sample collector was instructed to collect a total of 6 environmental samples, 2 samples in each of 3 pre-determined locations. The latter included (1) manure accumulation sites from lactating cow alleys or scraper lines; (2) manure storage areas such as lagoon, pits, or piles; and (3) if 2 or more cows were present, accumulated or concentrated manure from dry, calving, close-up, or sick pens. For each sample, the type of cows (lactating, dry, sick, or a combination) contributing to the sample, housing type (tiestall, freestall, loose housing, or other) and location (manure storage, exercise, and bedding pack, gutter, or alley) were recorded using a standardized description sheet. Any sample without a label was categorized as "not specified" and excluded from sample-type analysis. Each sample contained a subset of 4 samples that were thoroughly mixed before being stored on ice and mailed to the University of Calgary using Express Mail. All samples were processed within $7 \mathrm{~d}$ after collection using the TREK ESP II (TREK para-JEM, Trek Diagnostic Systems, Cleveland, $\mathrm{OH}$ ) culture protocol with subsequent F57-specific quantitative PCR (Mortier et al., 2014). Samples with a cycle threshold value $<40$ were categorized as MAP-positive.

\section{Statistical Analyses}

Analyses were conducted using $\mathrm{R}$ version 3.4 .2 ( $\mathrm{R}$ Core Team, Vienna, Austria) using the statistical R package "lme4" (Bates et al., 2015), and $P<0.05$ was considered significant.

The apparent herd-level MAP prevalence was estimated based on at least 1 environmental sample from a farm being MAP-positive. The true herd-level prevalence for each sampling period was calculated by adjusting apparent prevalence for imperfect sensitivity and specificity of the diagnostic test (Dohoo and Henrik, 2003). Sensitivity and specificity of a MAP test, based on 6 environmental samples, were previously estimated and were 0.69 and 0.99 , respectively (Wolf et al., 2014a).

The numbers of positive samples in $\mathrm{S} 1$ for farms that changed from positive to negative, $\mathrm{S} 1$ for farms that stayed positive, S2 for farms that changed from nega- tive to positive, and S2 for farms that stayed positive were compared using a Poisson regression model with a $\log$ link. Each of these 4 categories was included as a nominal predictor, with the outcome being number of MAP-positive environmental samples on the farm. Because this model assessed only records for farms classified as positive, the number of MAP-positive samples ranged from 1 to 6 . The model was estimated using the iteratively reweighted least squares algorithm.

To assess association of within-farm changes with herd size, 2 logistic regression models were used on subsets of the data. The first subset used only farms that tested positive in S1, with herd size as a predictor and S2 status as the outcome, whereas the second subset used only farms that tested negative in S1, with herd size as a predictor and S2 status as the outcome. This regression modeled the ratio of the odds of changing status in S2 to the odds of maintaining the same status in S2, conditional on herd status in S1. To determine the association between the number of MAP-positive samples per farm in S1 and test status in S2, logistic regression was conducted on a subset of the data that only included farms positive in S1. The outcome for this model was farm status in S2, with the only predictor being number of positive samples per farm in S1. This regression modeled the change in log odds of a herd testing MAP-positive in S2 per additional positive sample in S1.

The association between an environmental sample being MAP-positive based over the sampling periods and other predictor variables was assessed in 2 steps using mixed effect logistic regression. First, regression models were independently fit for each of 4 predictor variables: (1) type of cows (lactating, dry, sick, or combinations) from which the environmental sample was collected; (2) housing (tiestall, freestall, or loose housing); (3) location of sample collection (alley, gutter, or manure storage); and (4) number of adult cattle. Sampling period and an interaction of the predictor variable with sampling period were then forced into the model to allow for differences in temporal patterns within categories of other predictors. Coefficients involving sampling period can be interpreted as a measure of change between the 2 sampling periods, averaged across all herds in Saskatchewan. Variables significant at $P$ $\leq 0.05$ were included in the final model. Models were estimated by maximum likelihood where the likelihood function was estimated using 50 quadrature points and optimized using the limited-memory algorithm. In case of divergent models, they were updated for a further 2 $\times 10^{8}$ iterations using Nelder-Mead optimization until convergence was reached. Following convergence, Wald 95\% confidence intervals were calculated. 


\section{RESULTS}

\section{Prevalence}

True herd prevalence for all 167 farms sampled during S1, and 160 farms sampled during S2 was 77\% (95\% CI: $56-97 \%$ ) and $49 \%$ (95\% CI: 29-68\%), respectively. When only using the 148 farms that were sampled in both $\mathrm{S} 1$ and $\mathrm{S} 2$, in S1, 55\% of the Saskatchewan dairy farms had at least 1 MAP-positive environmental sample, resulting in a true herd prevalence estimate of $79 \%$ (95\% CI: 59-99\%). In S2, the proportion of herds with at least 1 MAP-positive environmental sample was $34 \%$, resulting in the true herd prevalence estimate of $48 \%$ (95\% CI: $26-69 \%$ ), a decrease compared with S1 $(P=0.03)$. A total of $104(70 \%)$ of farms sampled both in S1 and S2 did not change status (Table 1). Overall, MAP-negative farms in S1 were more likely to stay negative in S2, whereas S1-positive farms stay test positive more frequently in $\mathrm{S} 2$ than test negative (Table 1). Of farms that were MAP-negative in S1, those with a herd size of 51 to 100 or 101 to 150 cows were more likely to stay MAP-negative than change to positive (Table 1). Of farms that were MAP-positive in S2, herds of sizes 101 to 150 cows and >200 cows were more likely to remain positive (Table 1 ).

Thirty-one (21\%) farms that were MAP-positive in $\mathrm{S} 1$ but negative in S2 had, on average, 2.3 MAPpositive environmental samples of the 6 collected in S1, which was less than the average 3.0 samples on farms that stayed positive in $\mathrm{S} 2(P=0.01)$. The $13(9 \%)$ farms that were MAP-negative in $\mathrm{S} 1$ and positive in $\mathrm{S} 2$ had on average 1.8 positive samples in $\mathrm{S} 2$, which was not different from those positive only in $\mathrm{S} 1(P=$ 0.33). Among farms that had at least 1 MAP-positive environmental sample in S1, each additional MAP- positive sample reduced the odds of testing negative in $\mathrm{S} 2(\mathrm{OR}=-0.41 ; 95 \% \mathrm{CI}=0.16$ to $-2.53 ; P=0.01)$. For farms with at least 1 MAP-positive environmental sample in either sampling period, mean number of positive samples per farm did not change between S1 (2.9) and $\mathrm{S} 2(2.8 ; P=0.63)$.

\section{Characteristics of Environmental Samples}

Environmental samples in S1 and S2 were most frequently collected from locations containing lactating cows, from freestall pens, and from alleys (Table 2). When analyzing MAP-positive samples independently within characteristic type (cow group, housing, location, or herd size), differences were detected in proportions of positive samples among lactating, dry, sick, and combination cows in S1 (Table 2). Additionally, a smaller proportion of samples collected from loose housing were MAP-positive than samples from freestall housing in S1 and S2, and tiestalls had a fewer proportion of positive samples in S2 (Table 2). In both S1 and $\mathrm{S} 2$, the proportion of bedding packs samples were less often positive than samples collected from the alley (Table 2.) In both sampling periods, herds with $>200$ lactating cows had a higher odds of testing positive compared with herds $<51$ cows (Tables 2 and 3).

All environmental samples, regardless of characteristics (cow group, housing, or location) had decreased odds of culturing MAP-positive in S2 compared with $\mathrm{S} 1$; however, the largest decrease in odds of testing MAP-positive was from samples collected from dry cow areas (Tables 2 and 3). When analyzing all sample characteristics together, the decrease in the odds of being MAP-positive from S1 to S2 occurred across all sample characteristics; no differences were observed

Table 1. Mycobacterium avium ssp. paratuberculosis (MAP) status of 148 Saskatchewan dairy herds at the 2 sampling periods (categorized by herd size)

\begin{tabular}{|c|c|c|c|c|c|c|c|}
\hline $\mathrm{S} 1^{1}$ & $\mathrm{~S} 2^{1}$ & \multicolumn{5}{|c|}{ Adult cow herd size } & $\begin{array}{c}\text { All } \\
\text { No. }(\%)\end{array}$ \\
\hline \multirow[t]{2}{*}{ Negative } & Negative & $6(100)$ & $23(79)$ & $17(77)$ & $9(100)$ & $2(50)$ & $57(81)$ \\
\hline & Positive & $0(0)$ & $6(21)^{3}$ & $5(23)^{3}$ & $0(0)$ & $2(50)$ & $13(19)^{4}$ \\
\hline Positive & Negative & $5(83)$ & $11(50)$ & $4(29)$ & $6(43)$ & $5(22)^{3}$ & $31(40)^{5}$ \\
\hline
\end{tabular}

${ }^{1} \mathrm{~S} 1$ = sampling period $1 ; \mathrm{S} 2=$ sampling period 2.

${ }^{2}$ Percentage of farms with this MAP status within MAP status group S1.

${ }^{3}$ Different $(P<0.05)$ from number of farms that did not change status from S1 to S2 within the same herd size and S1 status.

${ }^{4}$ Different $(P<0.05)$ from number of farms that did not change status from S1 to S2 across herds of all sizes.

${ }^{5}$ Tendency for difference $(0.05>P>0.10)$ from number of farms that did not change status from S1 to S2 across herds of all sizes.

${ }^{6}$ Odds of changing status from S1 to S2 different $(P<0.05)$ from odds of changing status in herds of size 1 to 50 . 
among housing types or locations within the herd. However, herds with $>200$ cows had increased odds of remaining positive than herds with $<51$ cows (Table $3)$. The within-herd variance in the final mixed effects logistics regression was 3.75 (Table 3 ).

\section{DISCUSSION}

Based on environmental sampling, the prevalence of MAP-infected Saskatchewan dairy herds decreased in the 3.5-yr interval between the 2 sampling periods. All sample types were less frequently MAP-positive in S2 compared with $\mathrm{S} 1$, regardless of cow group contributing to the sample, housing type where the sample was collected, or location of collection. The largest decrease in the odds of being positive in S2 samples was among dry cows. Large herds (>200 cows) were more often MAPpositive than the smallest herds. Overall, prevalence of positive environmental samples decreased across all areas sampled within farms and was not specific to environmental sample characteristics.

Following the first sampling period, MAP-positive farms were offered whole-herd testing, and a risk assessment to be completed on farm, a protocol consis- tent with several control programs (Wolf et al., 2014b; Barkema et al., 2018). It has been reported that participation in control programs will only lead to an observable effect after 4 to $5 \mathrm{yr}$ (Nielsen and Toft, 2011). However, based on milk ELISA, decreases occurred in MAP prevalence at both herd and animal levels after only $2 \mathrm{yr}$ of participation in a control program (Verdugo et al., 2015). It is important to note that the decrease in herd prevalence for the current study was based on environmental samples, which have been reported as identifying a higher proportion of farms as MAP-positive than pooled fecal testing, or ELISA testing, despite the high correlation to prevalence estimates based on these methods (Berghaus et al., 2006).

Infection status of a herd was based on at least 1 MAP-positive environmental sample; however, the number of positive samples detected can provide a tool for estimating within-herd prevalence and monitoring how it changes from S1 to S2 (Pillars et al., 2009; Donat et al., 2015). Herds that changed from MAP-negative to positive had an average of 1.8 positive samples, whereas those that went from positive to negative had an average of 2.3 positive samples. Additionally, each added positive sample reduced the odds that a farm would

Table 2. Percentage of Mycobacterium avium ssp. paratuberculosis-positive (MAP-pos.) environmental samples collected from different locations on farm and herd sizes, during 2 sampling periods, $3.5 \mathrm{yr}$ apart

\begin{tabular}{|c|c|c|c|c|}
\hline Item & \multicolumn{2}{|c|}{ Aug. 2012-Oct. 2013 (S1) } & \multicolumn{2}{|c|}{ Sep. 2015-Feb. 2017 (S2) } \\
\hline \multicolumn{5}{|l|}{ Cow group } \\
\hline Lactating cows $^{2}$ & 632 & 28 & 552 & $25^{4}$ \\
\hline Dry cows & 128 & $20^{3}$ & 177 & $6^{3,4,5}$ \\
\hline Sick cows & 18 & $11^{3}$ & 7 & 14 \\
\hline Combination & 46 & $17^{3}$ & 62 & $6^{3}$ \\
\hline Freestall $^{2}$ & 479 & 30 & 451 & $25^{4}$ \\
\hline Tiestall & 64 & 11 & 68 & $4^{3}$ \\
\hline Loose housing & 279 & $23^{3}$ & 313 & $14^{3}$ \\
\hline Other & 0 & 0 & 1 & 1 \\
\hline Not specified & 66 & 21 & 55 & 9 \\
\hline \multicolumn{5}{|l|}{ Location } \\
\hline Alley $^{2}$ & 455 & 30 & 449 & $26^{4}$ \\
\hline$<51^{2}$ & 72 & 13 & 72 & 6 \\
\hline $51-100$ & 306 & 20 & 306 & 15 \\
\hline $101-150$ & 216 & 18 & 216 & 14 \\
\hline $151-200$ & 138 & 30 & 138 & 21 \\
\hline$>200$ & 156 & $51^{3}$ & 156 & $37^{3}$ \\
\hline
\end{tabular}

${ }^{1}$ Number of samples collected in total each sampling period. For herd size, number of farms.

${ }^{2}$ Reference category used as baseline in logistic regression model for that variable.

${ }^{3}$ Different $(P<0.05)$ from reference within sampling period.

${ }^{4}$ Different $(P<0.05)$ from $\mathrm{S} 1$ within the same category.

${ }^{5}$ Magnitude of change between S1 and S2 is different $(P<0.05)$ from reference. 
Table 3. Final mixed effects logistic regression model on the association between Mycobacterium avium ssp. paratuberculosis (MAP) environmental sample culture results and sample type in 148 Saskatchewan dairy herd samples in 2 sample periods

\begin{tabular}{|c|c|c|c|c|c|}
\hline Predictor/parameter & Coefficient $^{1}$ & SEM & $95 \% \mathrm{CI}^{2}$ & $P$-value & $\mathrm{OR}^{3}$ \\
\hline Lactating cows (intercept) ${ }^{4}$ & -2.21 & 0.75 & -3.68 to -0.74 & 0.003 & - \\
\hline Sick cows & -1.67 & 0.91 & -3.46 to 0.12 & 0.067 & 0.19 \\
\hline Combination & -1.01 & 0.57 & -2.13 to 0.10 & 0.074 & 0.36 \\
\hline \multicolumn{6}{|l|}{ Interaction } \\
\hline Sample period $2 \times$ lactating cows & -0.43 & 0.18 & -0.78 to -0.09 & 0.014 & - \\
\hline Sample period $2 \times$ combination & -0.40 & 0.84 & -2.05 to 1.25 & 0.634 & - \\
\hline \multicolumn{6}{|l|}{ Housing } \\
\hline Freestall & Referent $^{4}$ & & & & - \\
\hline Tiestall & -1.22 & 0.71 & -2.62 to 0.18 & 0.088 & 0.30 \\
\hline Loose housing & 0.19 & 0.37 & -0.54 to 0.92 & 0.611 & 1.21 \\
\hline Other $^{5}$ & -14.38 & - & - & - & $5.68 \times 10^{-7}$ \\
\hline \multicolumn{6}{|l|}{ Location sampled } \\
\hline$<51$ & Referent $^{4}$ & & & & - \\
\hline $51-100$ & 0.48 & 0.80 & -1.1 to 2.05 & 0.553 & 1.62 \\
\hline $101-150$ & 0.02 & 0.84 & -1.63 to 1.66 & 0.985 & 1.02 \\
\hline $151-200$ & 1.03 & 0.87 & -0.68 to 2.73 & 0.239 & 2.80 \\
\hline$>200$ & 2.50 & 0.85 & 0.84 to 4.16 & 0.003 & 12.18 \\
\hline Within-herd variance & 3.75 & 1.94 & & & \\
\hline
\end{tabular}

${ }^{1}$ Untransformed coefficients estimated by the model (log odds).

${ }^{2} 95 \%$ Wald CI.

${ }^{3}$ Transformed odds ratios (OR) from coefficients: OR $<1$ indicates a sample from the reference category is more likely to test MAP-positive than the comparator; OR $>1$ indicates a sample from the comparator is more likely to test MAP-positive than the reference category. Missing values represent coefficients that cannot be interpreted as OR.

${ }^{4}$ Baseline group against which others were compared was lactating cows in sampling period 1 , in freestall herds of size $<51$ cows where the sample was collected from an alley. Between sampling period differences are assumed common to all variables except cow group based on models 1 to 4 . ${ }^{5}$ Not enough herds used "other" housing to allow for accurate estimation of SEM.

test negative in S2. Therefore, it is likely that farms that became MAP-negative in S2 had a lower withinherd prevalence than the farms that remained positive (Raizman et al., 2004; Donat et al., 2015; Verdugo et al., 2015).

Differences of being MAP-positive in $\mathrm{S} 1$ or $\mathrm{S} 2$ were present within cow-group when assessing sample characteristics independently; however, these differences disappeared when all characteristics were considered together in 1 model. This was likely due to confounding, as certain characteristics are likely highly associated with each other (e.g., tiestall herds are smaller than freestall or bedded-pack herds), in addition to the wide range of number of samples that are included in each group. All sample types were less often positive in $\mathrm{S} 2$ than in S1; however, it should be noted that the decrease in proportions of positive samples was present among the groups that had the largest number of samples collected. The only characteristic that was different in S2 than S1 when considering characteristics independently, or together, was the larger decrease in the odds of MAP-positive samples in dry cow samples than in other groups. Most control programs have 2 main aims for controlling within-herd spread of infection: (1) preventing new infections of newborn calves, and (2) reducing number of infected animals shedding MAP (McKenna et al., 2006). Therefore, because wholeherd individual testing was offered to environmental sample-positive farms, it was likely that MAP-positive cows were identified in many herds, and that most of these cows were removed from the herd, based on current recommendations in Canadian control programs (McKenna et al., 2006; Cho et al., 2013), resulting in the change observed in S2. In Canada, calving is not seasonal, and dry cow groups are therefore always smaller than lactating cow groups. When within-herd prevalence decreases, the odds that at least 1 cow sheds MAP in the smaller group of dry cows will therefore decrease more than in the larger group of lactating cows.

Largest herds were more likely to test positive than smaller herds in both S1 and S2. This was consistent with findings that environmental samples collected from 
larger herds are more likely positive, and it is more difficult to decrease within-herd MAP prevalence in large herds over time (Wells and Wagner, 2000; Pillars et al., 2009). Although prevalence decreased in herds of all sizes, the majority of herds with $>200$ cows tested positive at both sampling periods. Alternatively, more medium-sized herds, categories 51-100 and 100-151, changed status from S1 to S2, with the majority of the smallest herds testing negative both times. This may support previous findings that herd size affects the ability to control MAP infection, and it should be taken into consideration for implementation and surveillance of future control programs.

One of the limitations of the current study is the lack of information (due to privacy reasons) regarding which farms participated in the whole-herd sampling, and whether changes were made based on suggestions outlined by the risk assessment. Currently, the authors can only speculate as to possible explanations for the decreased prevalence that was observed, based on control programs at the time of the study.

Additionally, based on the level of within-herd variance, unaccounted variables between farms contributed to differences (Table 3). Although this variance was accounted for in the model, this variance indicated that other influences, aside from environmental sample characteristics, were not accounted for but affected prevalence estimates.

\section{CONCLUSIONS}

Seventy percent of the farms did not change status between S1 and S2; therefore, the large decrease in the estimated herd-level MAP prevalence was due to a few farms testing positive in S1 and changing to negative status in S2, which was not specific to herd size. For farms with at least 1 positive sample in S1, each additional positive sample decreased the odds of testing negative in S2. All environmental samples regardless of type had decreased odds of testing positive in S2, with the largest decrease in environmental samples collected from areas containing dry cows. Herds $>200$ cows had increased odds of testing positive in at least 1 environmental sample, when compared with the smallest herds.

\section{ACKNOWLEDGMENTS}

This work was supported by the Natural Sciences and Engineering Research Council of Canada (NSERC) Industrial Research Chair in Infectious Diseases of Dairy Cattle. The NSERC Industrial Research Chair in Infectious Diseases of Dairy Cattle is supported by NSERC; Alberta Milk (Edmonton, AB, Canada); Dairy Farmers of Canada (Ottawa, ON, Canada); Dairy Farmers of Manitoba (Winnipeg, MB, Canada); Westgen Endowment Fund (Abbotsford, BC, Canada); BC Dairy (Burnaby, BC, Canada); CanWest DHI (Guelph, ON, Canada); and Canadian Dairy Network (Guelph, ON, Canada). The authors also thank the Saskatchewan Ministry of Agriculture and SaskMilk for their help contacting and collecting samples from dairy herds and providing funding for testing environmental samples, and Aaron Lucko (University of Calgary, Calgary, AB, Canada) for technical assistance processing samples in the laboratory.

\section{REFERENCES}

Barkema, H. W., K. Orsel, S. S. Nielsen, A. P. Koets, V. Rutten, J. P. Bannantine, G. P. Keefe, D. F. Kelton, S. J. Wells, R. J. Whittington, C. G. Mackintosh, E. J. Manning, M. F. Weber, C. Heuer, T. L. Forde, C. Ritter, S. Roche, C. S. Corbett, R. Wolf, P. J. Griebel, J. P. Kastelic, and J. De Buck. 2018. Knowledge gaps that hamper prevention and control of Mycobacterium avium subspecies paratuberculosis infection. Transbound. Emerg. Dis.65(Suppl 1):125-148. https://doi.org/10.1111/tbed.12723.

Bates, D., M. Mächler, B. Bolker, and S. Walker. 2015. Fitting linear mixed-effects models using lme4. J. Stat. Softw. 67. https://doi .org/10.18637/jss.v067.i01.

Berghaus, R. D., T. B. Farver, R. J. Anderson, C. C. Jaravata, and I. A. Gardner. 2006. Environmental sampling for detection of Mycobacterium avium spp. paratuberculosis on large California dairies. J. Dairy Sci. 89:963-970.

Cho, J., L. W. Tauer, Y. H. Schukken, R. L. Smith, Z. Lu, and Y. T. Gröhn. 2013. Cost-effective control strategies for Johne's disease in dairy herds. Can. J. Agric. Econ. 61:583-608.

Collins, M. T., V. Eggleston, and E. J. Manning. 2010. Successful control of Johne's disease in nine dairy herds: Results of a six-year field trial. J. Dairy Sci. 93:1638-1643.

Dohoo, I. R., and W. M. Henrik. 2003. Screening and diagnostic tests. Pages 93-115 in Veterinary Epidemiological Research. M. S. McPike, ed. Atlantic Veterinary College, Charlottetown, PE, Canada.

Donat, K., J. Kube, J. Dressel, E. Einax, M. Pfeffer, and K. Failing. 2015. Detection of Mycobacterium avium subspecies paratuberculosis in environmental samples by faecal culture and real-time PCR in relation to apparent within-herd prevalence as determined by individual faecal culture. Epidemiol. Infect. 143:975-985.

Kalis, C. H., J. W. Hesselink, H. W. Barkema, and M. T. Collins. 2001. Use of long-term vaccination with a killed vaccine to prevent fecal shedding of Mycobacterium avium subsp paratuberculosis in dairy herds. Am. J. Vet. Res. 62:270-274.

Lombard, J. E. 2011. Epidemiology and economics of paratuberculosis. Vet. Clin. North Am. Food Anim. Pract. 27:525-535.

Marcé, C., P. Ezanno, M. F. Weber, H. Seegers, D. U. Pfeiffer, and C. Fourichon. 2010. Invited review: modeling within-herd transmission of Mycobacterium avium subspecies paratuberculosis in dairy cattle: a review. J. Dairy Sci. 93:4455-4470.

McKenna, S. L., G. P. Keefe, A. Tiwari, J. A. VanLeeuwen, and H. W. Barkema. 2006. Johne's disease in Canada Part II: Disease impacts, risk factors, and control programs for dairy producers. Can. Vet. J. 47:1089-1099.

Mitchell, R. M., Y. Schukken, A. Koets, M. Weber, D. Bakker, J. Stabel, R. H. Whitlock, and Y. Louzoun. 2015. Differences in intermittent and continuous fecal shedding patterns between natural and experimental Mycobacterium avium subspecies paratuberculosis infections in cattle. Vet. Res. 46:66.

Mortier, R., H. W. Barkema, K. Orsel, R. Wolf, and J. De Buck. 2014 Shedding patterns of dairy calves experimentally infected with Mycobacterium avium subspecies paratuberculosis. Vet. Res. 45:71. 
Nielsen, S. S., and N. Toft. 2011. Effect of management practices on paratuberculosis prevalence in Danish dairy herds. J. Dairy Sci. 94:1849-1857.

Pillars, R., D. Grooms, and J. B. Kaneene. 2009. Longitudinal study of the distribution of Mycobacterium avium ssp. paratuberculosis in the environment of dairy herds in the Michigan Johne's disease control demonstration herd project. Can. Vet. J. 50:1039-1046.

Raizman, E. A., S. J. Wells, S. M. Godden, R. F. Bey, M. J. Oakes, D. C. Bentley, and K. E. Olsen. 2004. The distribution of Mycobacterium avium ssp. paratuberculosis in the environment surrounding Minnesota dairy farms. J. Dairy Sci. 87:2959-2966.

Raizman, E. A., S. J. Wells, S. M. Godden, J. Fetrow, and J. M. Oakes. 2007. The associations between culling due to clinical Johne's disease or the detection of Mycobacterium avium ssp. paratuberculosis fecal shedding and the diagnosis of clinical or subclinical diseases in two dairy herds in Minnesota, USA. Prev. Vet. Med. 80:166-178.

Smith, R. L., M. A. Al-Mamun, and Y. T. Gröhn. 2017. Economic consequences of paratuberculosis control in dairy cattle: A stochastic modeling study. Prev. Vet. Med. 138:17-27.

Sorge, U. S. K. Lissemore, A. Godkin, J. Jansen, S. Hendrick, S. Wells, and D. F. Kelton. 2011. Changes in management practices and apparent prevalence on Canadian dairy farms participating in a voluntary risk assessment-based Johne's disease control program. J. Dairy Sci. 94:5227-5237.

Tiwari, A., J. A. VanLeeuwen, S. L. McKenna, G. P. Keefe, and H. W. Barkema. 2006. Johne's disease in Canada Part I: Clinical symptoms, pathophysiology, diagnosis, and prevalence in dairy herds. Can. Vet. J. 47:874-882.

Verdugo, C., N. Toft, and S. S. Nielsen. 2015. Within- and betweenherd prevalence variation of Mycobacterium avium ssp. paratu- berculosis infection among control programme herds in Denmark (2011-2013). Prev. Vet. Med. 121:282-287.

Wells, S., and B. A. Wagner. 2000. Herd-level risk factors for infection with Mycobacterium paratuberculosis in US dairies and association between familiarity of the herd manager with the disease or prior diagnosis of the disease in the herd and use of preventive measures. J. Am. Vet. Med. Assoc. 216:1450-1457.

Whitlock, R. H. 2010. Paratuberculosis control measure in the USA. Pages 319-329 in Paratuberculosis-Organism, Disease and Control. M. A. Behr and D. M. Collins, ed. CABI, Wallingford, UK

Whittington, R. J., D. J. Marshall, P. J. Nicholls, I. B. Marsh, and L. A. Reddacliff. 2004. Survival and Dormancy of Mycobacterium avium ssp. paratuberculosis in the Environment. Appl. Environ. Microbiol. 70:2989-3004.

Wolf, R., H. W. Barkema, J. De Buck, and K. Orsel. 2015. Sampling location, herd size, and season influence Mycobacterium avium ssp. paratuberculosis environmental culture results. J. Dairy Sci. $98: 275-287$

Wolf, R., H. W. Barkema, J. De Buck, M. Slomp, J. Flaig, D. Haupstein, C. Pickel, and K. Orsel. 2014a. High herd-level prevalence of Mycobacterium avium subspecies paratuberculosis in Western Canadian dairy farms, based on environmental sampling. J. Dairy Sci. 97:6250-6259.

Wolf, R., F. Clement, H. W. Barkema, and K. Orsel. 2014b. Economic evaluation of participation in a voluntary Johne's disease prevention and control program from a farmer's perspective-The Alberta Johne's Disease Initiative. J. Dairy Sci. 97:2822-2834. 